\title{
The Simulation Study Of Transport System For Earth Work
}

\section{Allocation Of Rock-fill Dam}

\author{
XiaohangMa ${ }^{1, a}, \mathrm{KeLi}^{2, \mathrm{~b}}$ \\ ${ }^{1,2}$ Academy of Civil Engineering \& Architecture, Nanyang Normal University, Nanyang 473061, \\ China \\ ${ }^{a}$ email, ${ }^{b}$ email
}

Keywords: Rock-fill dam, Traffic and transportation system, System simulation, Object-oriented technology

\begin{abstract}
Based on the analysis of the traffic and transportation system in rock-fill dam, the method of modeling and simulation for the system with computer is proposed. For this, some technologies are applied, for example, the theory of the discrete event system simulation, object-oriented technology, the method of queuing theory and the network models. Finally testify the feasibility of earthwork allocation balance program by calculating stats.
\end{abstract}

\section{Introduction}

Material content balance problem is one of the key issues in rock-fill dam construction, relates to all aspects of the construction, affects the reasonable realization of the three goals --construction schedule, quality, investment. The road of the construction site is one of the main factors to solve the problem of earthwork balance, also it's an important guarantee that project completes successfullyon schedule.Transportation is a highly complex random dynamic processthat difficult to analyze through the simple mathematical analytical model, in this paper, the author uses the idea of discrete event system simulation combining with object-oriented technology, to realize the comprehensive simulation research of the road transportation system for rock-fill dam.Finally applying the simulation system we built to the practical engineeringto verify that the validity of the earthwork volumes allocation scheme by calculatingthe statistics of road transport, in the case of a designated earthwork allocation plan.

In the conditions of traditional modeling method, the road is only considered as a boundary condition for modeling, only get the average transport intensity of material, so we can't analysis the road transport conditions through the road, machinery and other related details of the results of the operation of the data.So the traditional method is difficult to guarantee the guiding significance of computing results for actual construction.In this paper, the author takes comprehensive simulation study on road transport system for rock-fill dam for the purpose of the overall simulation of road transportation condition by using the idea of discreteeventsystemsimulation and object-oriented technology combined with the basic idea of queuing theory and network model.

\section{Basic Theories and Concepts of System Simulation}

The Basic Theories and Concepts of Simulation Modeling. System simulation is building a simulation model that describes the structure and behavior of the real system and has a certain logic relationship or quantitative relationship for the purpose of system analysis based on the nature of 
the elements of the system and their relationship, to carry out a test or quantitative analysis, in order to obtain all kinds of information needed for the correct decisions. Simulation technology using the conceptual models instead of physical models, using conceptual models in computer operation instead of physical model in the trial operation, so it has good controllability, safety, repeatability and economic characteristics, etc.. The basic method of system simulation is to build the system structure model and quantitative analysis model, and convert it into the simulation model which is suitable for computer programming, and then carry on simulation experiment with the model. The system simulation method can be divided into continuous event system simulation and discrete event system simulation. Discrete event system simulation is closer to the practical background and application of engineering construction, it can grasp the system dynamic change process as long as only grasp each event occurrence time and describe the system state at different time according to the sequence of events without describe the specifics between two discrete times in details.

Discrete event system simulation in general, is to express the dynamic change process of the system through the advance of time or event. The simulation system is based on the simulation clock as the main line, looking for the time and the state of the event. There are two ways to advance the simulation clock, time step method and the event step method.

Methods of the Object-oriented. Object oriented is a method of understanding and abstracting the real world, which making the analysis and research of the system modeling and the human understanding of the objective world as same as possible. The oriented object simulation breaks through the traditional process-oriented simulation concept in theory, makes the modeling process close to the natural human being's way of thinking, the established model has inherent scalability and reusability, which is conducive to the establishment of visual modeling and simulation environment, so it provides a convenient way for large-scale complex system simulation analysis. In object oriented simulation, system is considered as a group of objects, the object is a separate entity, the attributes and the variation rules of object attributes known as the operation on the object is fully encapsulated within the object, the external effect put on the object must be conducted through the operation interface of the object. The running of the oriented object simulation system is implemented through the objects sending messages to each other. The oriented object simulation contains multi-type objects which were created by the corresponding class in the simulation run. There are several kinds:specific modeling object,abstract modeling object,random number and external input and message transfer.

\section{The Analysis and Design of Road Transport System for Rock-fill Dam}

The Analysis of Goal of System. The road transport system for rock-fill dam is a cyclical process made of a series of specific transport links, it can be divided into four main parts: loading, transit yard, unloading and roads(including the cross). The loading part contains material fields, transfer material field and excavation field; roads are divided into different sectionsaccordingto specificarrangementanduse; the unloading contains dam filling working face and transfer material field. The relationship of traffic flow between different objects as shown in fig. 1. 


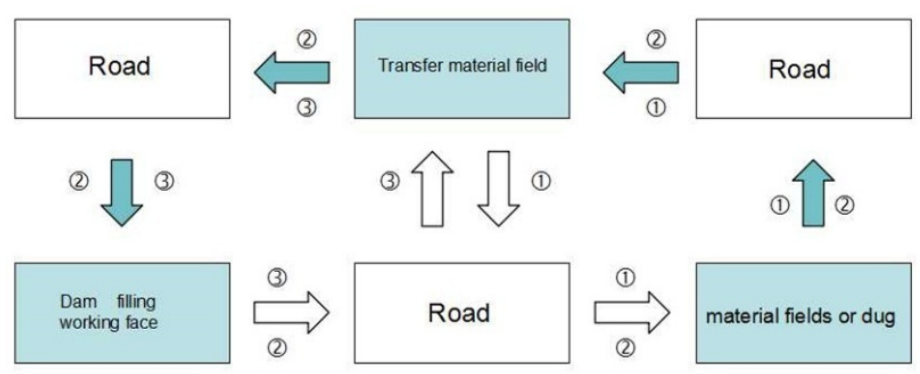

Fig. 1 Flow chart of the process of transportation

The solid block is the material handling work surface, the hollow box is object road; the solid arrow is heavy pulled into, the hollow arrow is empty return; direction (1) means the dump truck charged from the material fields or dug, loads to transit yard and returns to original loading place after unloading; direction (2) means the dump truck charged from the material fields or dug, unloadsat the workplace on the dam directly,then returns; direction (3)means the dump truck delivers the materials from the transfer material field to the dam face to unload.

According to the above analysis, the road transport system rock-fill dam construction has the following characteristics:a.There will be many material fields, slag fields and excavation objects, in order to achieve the shortest route, the system needs to compare the different driving routes in advance, so as to achieve the maximum efficiency of the automobile transportation, the space utilization of the road and the lowest comprehensive transportation cost. b. There will be many crosses in spot. The road conveying efficiency will appear to varying degrees of damage at the cross, so the traffic efficiency at the cross must be considered completely and studied by ways of modeling. c. At the start and end points of road (loading and unloading), there will always have queuing phenomenon caused by road congestion, the queuing phenomenon will reduce the utilization rate of vehicles relatively in time, so as to reduce the comprehensive transportation efficiency and cost of road transportation system. d. The regular change of operational process should be considered.

In order to make the road transport system meets the needs of project progress in the overall average, to meet the requirements of the construction intensity at peak period, also control the transportation cost, make utilization rate of roads and machinery etc. reach the maximum. To select the larger influence weight parameters: road and transport machinery as the Parameter index of basic data and technical measurement for the requirement of system modeling. Among these, road factors including road design level and intersection, transport machinery including mechanical types, configuration and operation condition.

Road transport system for rock-fill dam summarizes various parameters for system modeling and program evaluation, it is conducting an overall simulation of road transport process of rock-fill dam construction based on the characteristics and requirements of rock-fill dam project, combined with the influence analysis of road and mechanics. The optimization of road transport plan is mainly reflected in the following aspects: a.Traffic density and transport volume are in the range of the specification requirements, the shortest distance of comprehensive, and a balanced use of time utilization and space utilization of each road; b.A higher comprehensive utilization rate of transport machinery, including the loading and unloading time take a small proportion of the cycle time, the queue waiting time in the loading and unloading point is short, etc.; c.To make the transport machinery configuration and the proportion of transport machinery and loading machine reach optimal in the range of transport machinery speed requirements, also, to meet the two conditions in 
front.

System Model Division. Divide the road transportation system into three models: the road junction model, the queuing network model, the random function model.

a. The road junction model is to number the main road nodes, divide each main road into small sections, number each section with the characteristics of activity-on-arrow network and set up appropriate road attribute. In this model, the main constraint conditions are road length and road transport capacity.

b.The queuing service system is composed of the customer and the service desk. Any person or thing that is required to receive a service is called a customer, such as dump truck; any person or thing that gives a customer service is a service desk, such loader. A queuing system can be described abstractly as follows: customers come for services, if they can't get the service immediately and allow the queue to wait, they shall join the waiting group, then leave the system after getting the services.

c.Random probability distribution model can give a reasonable random number to the construction machinery and traffic time in the condition of given a probability distribution type has been set, so as to simulate the whole process. According to the previous study of random distribution of types we choose, this system uses normal distribution random variable types to simulate the loading time of the loader, uses the negative exponential distribution random variable types to simulate the unloading material discharging time.

\section{Design and Implementation of Simulation System}

Overall Design of System. The system is set in the earthwork of rock-fill dam, which takes the process of road transportation in the balanced earth-rock allocation as the simulation agent and simulates the working condition of the road transportation system all time. It gets a transport planning scheme which the transportation cost is the lowest and the efficiency is the highest, the use of resources achieves maximum, to achieve the purpose of the requirements of the construction schedule, through the comparison and analysis of various operating parameters and parameters. The system takes the form of three-tier architecture: user management layer, database management layer and model construction and calculation layer. Building basic database, in order to make the user input parameters of roads and vehicles in road transport system easily. When the system simulation is finished, the system will output the road traffic density, the heavy traffic, the operation rate of machinery and failure rate to the result database to facilitate the users to view.

The system disperses the road transport system first, decompose it into many forms with specific functions and features, according to the idea of discrete event system simulation and the object oriented design method of discrete event system simulation. In this system, the system is conceptualized as time management types, activity items, machinery, roads and environmental parameters, and these types can be decomposed into each subclass further as shown in fig. 2 . 


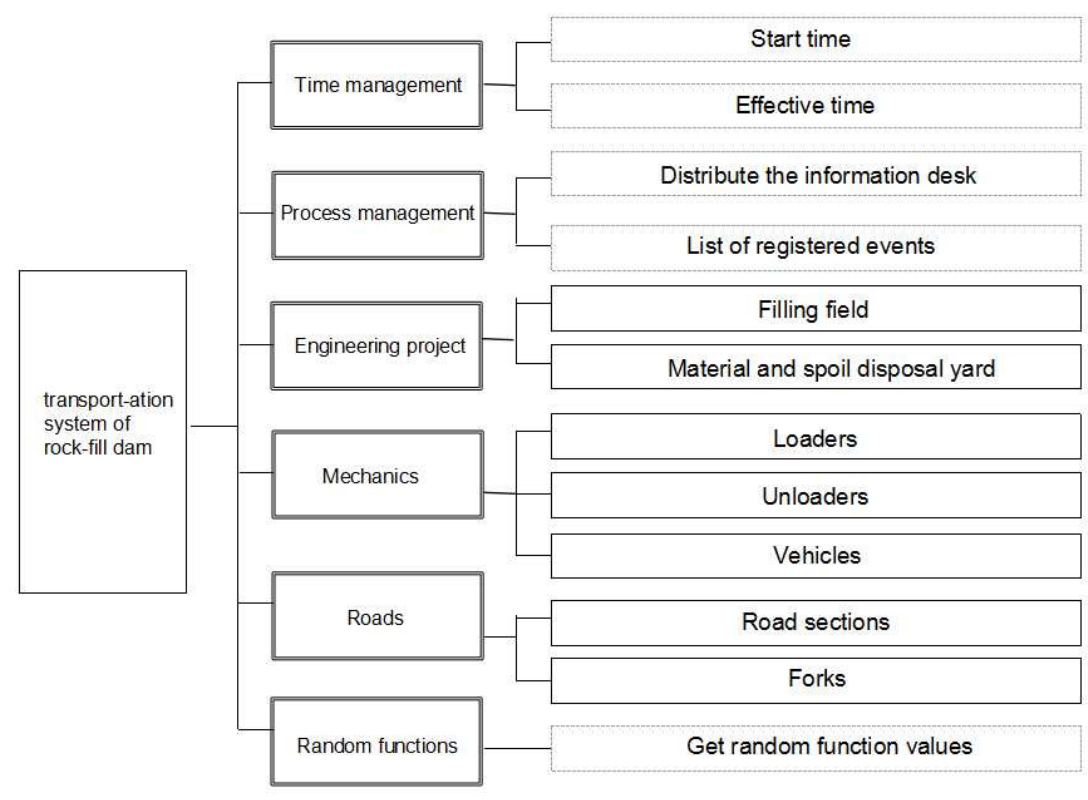

Fig. 2 Decomposition Diagram of Road Transportation System for Rock-fill Dam

Use the object-oriented implementation technology to encapsulate activity forms of each entity within the entity, set up the final discrete simulation system with message communication between functional models which built by message passing. To determine the generative mechanism and time of the next event according to the discrete event system simulation event table, start event mechanism when system time jumps to the event time, then activate the model until the end of the event, finally form the final simulation process according to the event jumps.

Simulation example. The size of a Water conservancy and Hydropower Engineering project is a large $\mathbb{I}$, and the engineering grade is second class. The gross amount of embankment material are about $7000000 \mathrm{~m}^{3}$, the quantity of excavation material can be used are about $600000 \mathrm{~m}^{3}$.

The dam body is divided into the major material zone and the minor material zone, an anti filter layer is set up at dam heel. The rock-fill materials in major material zone are limestone from ma-chang river quarry $3 \mathrm{~km}$ away, the rock-fill materials in minor material zone comes from gravel stone and spillway. To collect the initial conditions of simulation such as the construction schedule, the layout of the material yard, the road planning, the machinery condition, the working time and condition, etc. before simulating on the computer, then begin the simulation after entering the simulation parameters, part of results of simulation are shown in fig. 3. 


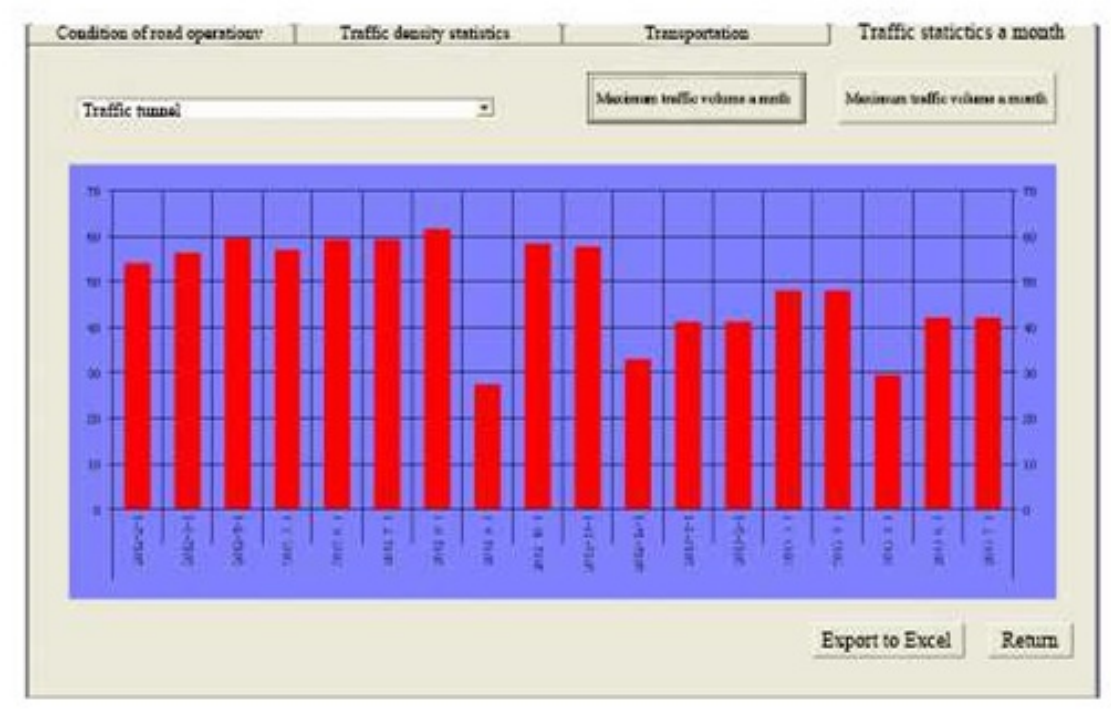

Figure 3 Maximum traffic density per-month in traffic tunnel

Analysis of calculation:a. Maximum traffic density is 62 trucks per-hour, and it's below the standard of secondary road-85 trucks per-hour in $<$ Specifications for the design of factory and mine roads $>$, b. The use of time of the main road is more uniform, the use of road is more uniform, the whole utilization is more uniform.

\section{Conclusion}

The system we designed that builds a road transportation system suitable for rock-fill dam construction, it is aimed at the shortage of transportation system simulation model in balanced earthwork allocation activities, it carries on an object oriented discrete analysis for the transportation combine with the characteristics of cut-fill transition, for the final purpose of truly reflecting the transport process. The system is of universal applicability, also it has some shortages, such as a simply consideration of a single type of loader in modeling, the simple and single output form.

\section{References:}

[1] Arcelik R, Besley M. Micro-simulation and analytical methods for modeling urban traffic. Paper presented at the Conference on Advance Modeling Techniques and Quality of Service in Highway Capacity Analysis[J]. Truckee, California, USA. July 2001

[2] Yang Jianguo, etc.. The Reality Basis and Reality-Based Modeling Methodology Applied in Traffic Micro Simulation Models. Proceeding of Asian Simulation Conference the 5th International Conference on System Simulation and Scientific Computing[J]. 2002,1:142-145.

[3] Patrick A.M.Ehlert, Leon J.M.Rothkrantz. Microscopic traffic simulation with reactive driving agents. 2001IEEE Intelligent Transportation Systems Conference Proceedings, August, 2001, $861 \sim 865$

[4]Sakda Panwai, Hussein Dia. Comparative evaluation of microscopic car-following behavior. Intelligent Transportation Systems, 2005, 6(3): 314 325

[5] Qi Yang, Haris N. Koutsopoulos, Moshe E. Ben-Akiva. A Simulation Laboratory for Evaluating Dynamic Traffic Management Systems. Prepared for Presentation at the $79^{\text {th }}$ Annual Meeting of Transportation Research Board, 1999 